\title{
ASYMPTOTIC EXPANSIONS AND NUMERICAL SIMULATIONS OF I-V RELATIONS VIA A STEADY STATE POISSON-NERNST-PLANCK SYSTEM
}

\author{
MINGJI ZHANG
}

\begin{abstract}
A steady state Poisson-Nernst-Planck (PNP) system is studied both analytically and numerically with particular attention on I-V relations of ion channels. Assuming the dielectric constant $\varepsilon$ is small, the PNP system can be viewed as a singularly perturbed system. Due to the special structures of the zeroth order inner and outer systems, one is able to derive more explicit expressions of higher order terms in asymptotic expansions. For the case of zero permanent charge, under the assumption of electro-neutrality at both ends of the channel, our result concerning the I-V relation for two oppositely charged ion species is that the third order correction is cubic in $V$, and, furthermore (Theorem 4.1), up to the third order, the cubic I-V relation has three distinct real roots (except for a very degenerate case) which corresponds to the bi-stable structure in the FitzHugh-Nagumo simplification of the Hodgkin-Huxley model. Three numerical experiments are conducted to check the cubic-like feature of the I-V curve, study the boundary value effect on the I-V relation and investigate the permanent charge effect on the $\mathrm{I}-\mathrm{V}$ curve, respectively.
\end{abstract}

1. Introduction. Ion channels are cylindrical, hollow proteins that regulate the movement of ions (mainly $\mathrm{K}^{+}, \mathrm{Na}^{+}, \mathrm{Ca}^{++}$and $\mathrm{Cl}^{-}$) across almost all biological membranes (see [9]). The most relevant properties of a channel are permeation and selectivity, and an important characterization is the current-voltage (I-V) relation. The I-V relation adopted in the FitzHugh-Nagumo simplification of the famous Hodgkin-Huxley

2010 AMS Mathematics subject classification. Primary 34A26, 34B16, 34D15, 37D10, 92C35.

Keywords and phrases. singular perturbation, matched asymptotic expansion, $\mathrm{I}-\mathrm{V}$ relations.

This work is supported by a Summer Research Scholarship from Research and Graduate Study, University of Kansas, and start-up funds for new faculties at New Mexico Tech.

Received by the editors on April 23, 2013, and in revised form on October 25, 2013. 
systems which describe the propagation of action potential of an ensemble of channels in a biological membrane is cubic-like. A natural question arising here is whether this cubic-like feature can be obtained from a single channel?

For a single channel model the most successful one is the PoissonNernst-Planck (PNP) theory (see $[\mathbf{2}, \mathbf{3}, \mathbf{4}, \mathbf{7}, \mathbf{1 9}]$, etc.). With the assumption that the channel is narrow, it can be effectively viewed as a one-dimensional channel and normalized as the interval $[0,1]$. A natural one dimensional steady-state PNP type model for ion flows of $n$ ion species is (see $[\mathbf{1 7}, \mathbf{1 9}]$ )

$$
\begin{aligned}
& \frac{1}{h(x)} \frac{d}{d x}\left(\varepsilon_{r}(x) \varepsilon_{0} h(x) \frac{d \Phi}{d x}\right)=-e\left(\sum_{j=1}^{n} z_{j} c_{j}(x)+Q(x)\right), \\
& \frac{d \mathcal{J}_{i}}{d x}=0, \quad-\mathcal{J}_{i}=\frac{1}{k T} D_{i}(x) h(x) c_{i}(x) \frac{d \mu_{i}}{d x}, \quad i=1,2, \ldots, n,
\end{aligned}
$$

where $e$ is the elementary charge, $k$ the Boltzmann constant, $T$ the absolute temperature; $\Phi$ is the electric potential, $Q(x)$ the permanent charge of the channel, $\varepsilon_{r}(x)$ the relative dielectric coefficient, $\varepsilon_{0}$ is the vacuum permittivity; $h(x)$ the area of the cross-section of the channel over the point $x$; for the $i$ th ion species, $c_{i}$ is the concentration, $z_{i}$ the valence (the number of charges per particle), $\mu_{i}$ the electrochemical potential, $\mathcal{J}_{i}$ the flux density and $D_{i}(x)$ the diffusion coefficient. The boundary conditions are, for $i=1,2, \ldots, n$,

$$
\Phi(0)=V, \quad c_{i}(0)=L_{i} ; \quad \Phi(1)=0, \quad c_{i}(1)=R_{i} .
$$

For a solution of systems (1.1) and (1.2), the rate of flow of charge through a cross-section or current $\mathcal{I}$ is:

$$
\mathcal{I}=\sum_{j=1}^{n} z_{j} \mathcal{J}_{j} .
$$

For fixed $L_{i}$ 's and $R_{i}$ 's, formula (1.3) provides a relation of the current $\mathcal{I}$ on the voltage $V$, which is the so-called $I-V$ relation (current-voltage relation). The rate of flow of matter through a cross-section $\mathcal{T}$ is given by:

$$
\mathcal{T}=\sum_{j=1}^{n} \mathcal{J}_{j} .
$$


In this work, we mainly focus on the I-V relation, more precisely, our main interest in the I-V relation is to derive the asymptotic expansion

$$
\mathcal{I}=I_{0}+\varepsilon I_{1}+\varepsilon^{2} I_{2}+\varepsilon^{3} I_{3}+\cdots .
$$

For consistency, we also write

$$
\mathcal{T}=T_{0}+\varepsilon T_{1}+\varepsilon^{2} T_{2}+\varepsilon^{3} T_{3}+\cdots
$$

It is known that, in general, the $\mathrm{I}-\mathrm{V}$ relation is not unique (see $[8,18,21,22,25,26]$ for $Q \neq 0$ and see [14] even for $Q=0$ when more ion species are involved). In Section 3, we will consider a special case where the I-V relation is indeed unique. For simplicity, in this work, we make the following assumptions:

(A1) Considering two ion species $(n=2)$ with $z_{1}=\alpha>0$ and $-\beta=z_{2}<0$.

(A2) Setting the permanent charge to be zero: $Q(x)=0$.

(A3) For the electrochemical potential $\mu_{i}$, considering the ideal component $\mu_{i}^{i d}$ only, which is given by

$$
\mu_{i}^{i d}(x)=z_{i} e \Phi(x)+k T \ln \frac{c_{i}(x)}{c_{0}} .
$$

(A4) Assuming the relative dielectric coefficient and the diffusion coefficient to be constants, that is, $\varepsilon_{r}(x)=\varepsilon_{r}$ and $D_{i}(x)=D_{i}$.

To further simplify the problem, we make the dimensionless re-scaling,

$$
\phi=\frac{e}{k T} \Phi, \quad \bar{V}=\frac{e}{k T} V, \quad \varepsilon^{2}=\frac{\varepsilon_{r} \varepsilon_{0} k T}{e^{2}}, \quad J_{i}=\frac{\mathcal{J}_{i}}{D_{i}} .
$$

The boundary value problem (1.1) and (1.2) then becomes

$$
\begin{aligned}
& \frac{\varepsilon^{2}}{h(x)} \frac{d}{d x}\left(h(x) \frac{d}{d x} \phi\right)=\beta c_{2}-\alpha c_{1}, \quad \frac{d J_{i}}{d x}=0, \\
& h(x) \frac{d c_{1}}{d x}+\alpha h(x) c_{1} \frac{d \phi}{d x}=-J_{1}, \\
& h(x) \frac{d c_{2}}{d x}-\beta h(x) c_{2} \frac{d \phi}{d x}=-J_{2},
\end{aligned}
$$

with the boundary conditions:

$$
\phi(0)=\bar{V}, \quad c_{i}(0)=L_{i} ; \quad \phi(1)=0, \quad c_{i}(1)=R_{i} .
$$


With the assumption that $\varepsilon$ is small, viewing it as the singular parameter, system (1.7) together with the boundary condition (1.8) will be treated as a singular boundary value problem. The general framework of the classical singular perturbation theory and the newly developed geometrical singular perturbation theory suggest studying the asymptotic expansion of the I-V relation.

For two types of ion species with $Q=0$ (see $[\mathbf{2 4}, \mathbf{2 3}]$ for a treatment using a three-dimensional PNP model), the zeroth order I-V relation is obtained explicitly (see $[4, \mathbf{9}, \mathbf{1 0}, \mathbf{1 3}, \mathbf{1 5}, \mathbf{1 6}]$, etc). For this case, the zeroth order I-V relation is linear while experimentally, the phenomenon of "saturation" of I-V relation is investigated. In [1], a one dimensional steady-state PNP system has been studied using asymptotic expansion approach with particular attention to the I-V relations. The result shows that:

- The first order correction to the zeroth order linear I-V relation is generally quadratic in $V$;

- when the electro-neutrality condition is enforced at both ends of the channel, there is no first order correction;

- the second order correction is cubic in $V$. Moreover, under electro-neutrality condition, up to the second order (in $\varepsilon$ ), the $\mathrm{I}-\mathrm{V}$ relation is a cubic function with three distinct real roots.

A natural question arising here is whether the higher order corrections follow this pattern? More precisely, is the third order correction quartic in $V$ ? What about the fourth order correction?

Our goal in this paper is to further examine higher order asymptotic expansions of the I-V relation following the idea in [1] and to provide answers to these interesting questions. For the special case mentioned above, the third order correction turns out to be cubic with the electroneutrality condition (see formula (3.7)) even though a quartic function is expected, which gives us the first surprise. Immediately, we get another interesting question: are the other higher order corrections also keeping this feature? This leads to the study of the fourth order correction. However, to our surprise, the fourth order correction is quintic (see formula (4.2)) instead of being cubic. Furthermore, for the third order correction, the coefficient of the cubic term is always negative except for a highly degenerate case (see Theorem 4.1, Lemma 4.3 and Lemma 4.4). An importance of this negative sign is 
that, up to the third order, the cubic I-V function has three distinct real roots-this agrees qualitatively with $\mathrm{I}-\mathrm{V}$ relation adopted in the FitzHugh-Nagumo simplification of the Hodgkin-Huxley systems. The existence of three distinct real roots of the $\mathrm{I}-\mathrm{V}$ relation is responsible for the bi-stable structure in the FitzHugh-Nagumo system.

Numerical simulations are performed for both the cases with zero permanent charge and nonzero one respectively. For the case with zero permanent charge, it allows us to make a comparison between the analytical results and our numerical results. And, meanwhile, one can investigate the effect of the boundary conditions on the I-V relations. For the one with nonzero permanent charge defined by

$$
Q(x)=\left\{\begin{array}{l}
0 \text { for } 0<x \leq a \\
Q \text { for } a<x<b \\
0 \text { for } b \leq x \leq 1
\end{array}\right.
$$

where $Q$ is a nonzero constant, we mainly focus on the cubic-like feature of the $\mathrm{I}-\mathrm{V}$ relation and the effect of the permanent charge.

A thorough study of higher order asymptotic expansion of $\phi$ and $c_{i}$ 's is necessary to obtain higher order asymptotic expansions of the $\mathrm{I}-\mathrm{V}$ relation. Both the geometric singular perturbation method and the classical matched asymptotic expansion method work well for the zeroth order term (see $[\mathbf{1}, \mathbf{4}, \mathbf{9}, \mathbf{1 0}, \mathbf{1 5}, \mathbf{1 6}]$ ) at least for the special case mentioned above (see $[\mathbf{8}, \mathbf{1 4}]$ for a treatment of general situations). For higher order terms, the classical matched asymptotic expansion approach are applied since it seems that a direct application of the geometric singular perturbation theory does not work, a research direction worthwhile to explore. It's well known that higher order terms satisfy linear but non-autonomous and non-homogeneous systems. The homogeneous parts of the linear systems are the same and are nothing but the linearizations of the zeroth order nonlinear system along the zeroth order (inner and outer) solutions. While, in general, it is impossible to get explicit solutions of a linear non-autonomous system, a special feature of the problem at hand that the zeroth order nonlinear system possesses a complete set of integrals and each integral provides an integral for the linearization (see Propositions 2.2 and 2.3) allows us to carry out a detailed asymptotic analysis.

The paper is organized as follows. In Section 2, we briefly restate the outer and inner systems for each order in the asymptotic expansions 
from [1], and the matching principle. Starting in Section 3, we restrict ourselves to the special case and examine the outer, inner expansions and matching. Previous results for lower order systems from [1] are briefly restated for completeness, and the third order expansions and matching are detailed under the electro-neutrality condition. In Section 4, under the electro-neutrality condition, we focus on the I$\mathrm{V}$ relation up to the third order in $\varepsilon$ and obtain our main result. In Section 5, numerical simulations are performed to system (5.3) with boundary condition (1.8) for both $Q(x)=0$ and $Q(x) \neq 0$, and corresponding I-V relation curves are obtained. Interesting phenomena are investigated.

2. Systems for asymptotic expansions. In this section, we apply the method of asymptotic expansions for both outer and inner systems to study the I-V relations of the PNP model discussed above. In the current context, the outer systems "determine" the dynamics of ion flows within the channel, and the inner systems "govern" the potential boundary layers that represents the effects of boundary conditions from the bath conditions. The matching principle then provides the intersection between the internal dynamics and the boundary conditions.

2.1. Outer systems for each order. In this section, we assume $Q$ is constant and look for outer expansion of the form, for $i=1,2$,

$$
\begin{aligned}
\phi(x ; \epsilon) & =\phi_{0}(x)+\epsilon \phi_{1}(x)+\epsilon^{2} \phi_{2}(x)+\cdots, \\
c_{i}(x ; \epsilon) & =c_{i 0}(x)+\epsilon c_{i 1}(x)+\epsilon^{2} c_{i 2}(x)+\cdots, \\
J_{i} & =J_{i 0}+\epsilon J_{i 1}+\epsilon^{2} J_{i 2}+\cdots .
\end{aligned}
$$

Substituting (2.1) into (1.7) and denoting the derivatives with respect to $x$ by overdots, with the convention that $\phi_{-1}=\phi_{-2}=0, \delta_{0}=1$ and $\delta_{j}=0$ for $j \neq 0$, upon introducing $u_{j}=\dot{\phi}_{j}$, the $j$ th order system in $\varepsilon$ is, for $i=1,2$,

$$
\begin{aligned}
\dot{\phi}_{j-2} & =u_{j-2}, \quad \dot{u}_{j-2}=-\left(\alpha c_{1 j}-\beta c_{2 j}+\delta_{j} Q\right), \\
\dot{c}_{i j} & =-\sum_{p+q=j}\left(\alpha c_{1 p}-\beta c_{2 p}\right) u_{q}-J_{i j} .
\end{aligned}
$$


Remark 2.1. An observation is that the homogeneous part for $c_{i j}$ 's is

$$
C_{j}^{\prime}=-u_{0}(x)\left(\begin{array}{cc}
\alpha & 0 \\
0 & -\beta
\end{array}\right)\left(\begin{array}{l}
c_{1 j} \\
c_{2 j}
\end{array}\right) .
$$

Once $u_{0}(x)$ is found, this system can be simply integrated. And, hence, system (2.2) can be solved.

\subsection{Inner systems for each order.}

2.2.1. Inner systems at the left boundary $x=0$. At the boundary $x=0$, in terms of the inner variable $\xi=x / \epsilon$, let $\Phi(\xi ; \epsilon)=\phi(\epsilon \xi ; \epsilon)$ and $C_{i}(\xi ; \epsilon)=c_{i}(\epsilon \xi ; \epsilon)$. System (1.7) becomes, for $i=1,2$,

$$
\begin{aligned}
& \frac{d^{2}}{d \xi^{2}} \Phi=-\left(\alpha C_{1}-\beta C_{2}+Q\right), \quad \frac{d J_{1}}{d \xi}=\frac{d J_{2}}{d \xi}=0 \\
& \frac{d C_{1}}{d \xi}+\alpha C_{1} \frac{d \Phi}{d \xi}=-\epsilon J_{1}, \quad \frac{d C_{2}}{d \xi}-\beta C_{2} \frac{d \Phi}{d \xi}=-\epsilon J_{2}
\end{aligned}
$$

We look for the inner expansion of the form:

$$
\begin{aligned}
\Phi(\xi ; \epsilon) & =\Phi_{0}(\xi)+\epsilon \Phi_{1}(\xi)+\epsilon^{2} \Phi_{2}(\xi)+\cdots \\
C_{i}(\xi ; \epsilon) & =C_{i 0}(\xi)+\epsilon C_{i 1}(\xi)+\epsilon^{2} C_{i 2}(\xi)+\cdots \\
J_{i} & =J_{i 0}+\epsilon J_{i 1}+\epsilon^{2} J_{i 2}+\cdots
\end{aligned}
$$

We have, by introducing $U_{j}=\Phi_{j}^{\prime}$,

$$
\begin{aligned}
\Phi_{j}^{\prime} & =U_{j}, \quad U_{j}^{\prime}=-\left(\alpha C_{1 j}-\beta C_{2 j}\right)-\delta_{j} Q, \\
C_{1 j}^{\prime} & =-\sum_{p+q=j} \alpha C_{1 p} U_{q}-J_{1(j-1)}, \\
C_{2 j}^{\prime} & =\sum_{p+q=j} \beta C_{2 p} U_{q}-J_{2(j-1)} .
\end{aligned}
$$

For $j=0$, the system is

$$
\begin{aligned}
\Phi_{0}^{\prime} & =U_{0}, \quad U_{0}^{\prime}=-\left(\alpha C_{10}-\beta C_{20}\right)-Q, \\
C_{10}^{\prime} & =-\alpha C_{10} U_{0}, \quad C_{20}^{\prime}=\beta C_{20} U_{0} .
\end{aligned}
$$

and, for all $j \geq 1$, the homogeneous part of (2.5) is the same, and it is the linearization of the zeroth order system (2.6). 
There is a specific structure of system (2.6) that, together with an abstract result, allows one to get a closed form for solutions of (2.5). The specific structure is

Proposition 2.2. The zeroth order inner system (2.6) has a complete set of (3) first integrals given by,

$$
H_{1}=C_{10} e^{\alpha \Phi_{0}}, \quad H_{2}=C_{20} e^{-\beta \Phi_{0}}, \quad H_{3}=\frac{1}{2} U_{0}^{2}-C_{10}-C_{20}+Q \Phi_{0} .
$$

Proof. This can be verified directly (see also [14]).

A crucial result whose proof is provided in [1] is given below.

Proposition 2.3. Consider an autonomous system

$$
z^{\prime}=f(z), \quad z \in \mathbb{R}^{m} .
$$

For a solution $z_{0}(t)$ of $(2.7)$, consider the linearization along $z_{0}(t)$ :

$$
Z^{\prime}=D f\left(z_{0}(t)\right) Z, \quad Z \in \mathbb{R}^{m} .
$$

If a $C^{2}$ function $H: \mathbb{R}^{m} \rightarrow \mathbb{R}$ is an integral of system (2.7) (that is, $H(z(t))$ is independent of $t$ for any solution $z(t)$ of (2.7), then $G(Z, t)=\left\langle\nabla H\left(z_{0}(t)\right), Z\right\rangle$ is an integral of the linear system (2.8) (that is, $G(Z(t), t)$ is independent of $t$ for any solution $Z(t)$ of $(2.8))$.

Noticing that the homogeneous part of (2.5) for $j \geq 1$ is the linearization of the zeroth order system (2.6), a complete set of integrals for the homogeneous part of (2.5) can be derived from Propositions 2.2 and 2.3. An application of variation of parameters allows one to get a closed form for the solutions of (2.5).

2.2.2. Inner systems at the right boundary $x=1$. In a similar way, at the right boundary $x=1$ in terms of the inner variable $\xi=(-1+x) / \epsilon$, and let $\Psi(\xi ; \epsilon)=\phi(1+\epsilon \xi ; \epsilon)$ and $D_{k}(\xi ; \epsilon)=c_{k}(1+\epsilon \xi ; \epsilon)$. By introducing 
$V_{j}=\Psi_{j}^{\prime}$, we get

$$
\begin{aligned}
\Psi_{j}^{\prime} & =V_{j}, \quad V_{j}^{\prime}=-\left(\alpha D_{1 j}-\beta D_{2 j}\right)-\delta_{j} Q, \\
D_{1 j}^{\prime} & =-\sum_{p+q=j} \alpha D_{1 p} V_{q}-J_{1(j-1)}, \\
D_{2 j}^{\prime} & =-\sum_{p+q=j} \beta D_{2 p} V_{q}-J_{2(j-1)} .
\end{aligned}
$$

The same observation for inner systems at $x=0$ applies here.

Remark 2.4. For a more general derivation of the outer and inner systems, one can read [1].

2.3. Asymptotic matching principle. The piecing of the inner solution and outer solution is achieved by matching principles. In this work, we will use the asymptotic matching principle of Van Dyke (see $[5,6,12])$ for our matching purpose. With a suitable hypothesis, the principle can be rigorously justified. It turns out that, for the problem handled in this paper, the so-called outer manifold is normally hyperbolic [8, 14], and Van Dyke's principle of asymptotic matching is justified (see, for example, [20]).

To state it, we use the notion of expansion operators in [12]: for a function $g(x ; \epsilon)$ in terms of the outer variable $x$ and a function $f(\xi ; \epsilon)$ in terms of the inner variable $\xi$, the $k$ th order outer and inner expansions are denoted by, respectively,

$$
E_{x}^{k}(g(x ; \epsilon))=\sum_{j=0}^{k} \epsilon^{j} g_{j}(x), \quad E_{\xi}^{k}(f(\xi ; \epsilon))=\sum_{j=0}^{k} \epsilon^{j} f_{j}(\xi) .
$$

The $k$ th order matching principle to be applied is $E_{x}^{k} E_{\xi}^{k}(f)=$ $E_{\xi}^{k} E_{x}^{k}(g)$ in terms of either the outer variable $x$ or the inner variable $\xi$.

For our problem, we will apply the matching principle to $(\phi(x ; \epsilon)$, $\left.c_{k}(x ; \epsilon)\right)$ and $\left(\Phi(\xi ; \epsilon), C_{k}(\xi ; \epsilon)\right)$ at the left boundary $x=0$ and to $\left(\phi(x ; \epsilon), c_{k}(x ; \epsilon)\right)$ and $\left(\Psi(\xi ; \epsilon), D_{k}(\xi ; \epsilon)\right)$ at the right boundary $x=1$.

3. Third order matching under electro-neutrality conditions. In this section, with $\alpha=\beta=1$, under the electro-neutrality 
assumption $L_{1}=L_{2}=L$ and $R_{1}=R_{2}=R$, we will derive the matched asymptotic expansions for the third order over the interval $[0,1]$ and, through matching, we establish the third order correction.

For completeness, we summarize the results for lower order asymptotic expansions from [1] as follows:

Theorem 3.1. If $L \neq R$, under the electro-neutrality condition, with $I_{k}=J_{1 k}-J_{2 k}$ and $T_{k}=J_{1 k}+J_{2 k}, k=0,1,2$, for the outer system, we have

- Zeroth order: $\phi_{0}(x)=b_{0}+\left(I_{0} / T_{0}\right) \ln \left|a_{0}-T_{0} x\right|, c_{10}(x)=$ $c_{20}(x)=\left(a_{0}-T_{0} x\right) / 2$.

- First order: $\phi_{1}(x)=c_{11}(x)=c_{21}(x)=0$.

- Second order: $c_{12}(x)=\left(a_{2}-T_{2} x\right) / 2+\left(I_{0}^{2}+2 I_{0} T_{0}\right) / 4\left(a_{0}-T_{0} x\right)^{2}$, $c_{22}(x)=\left(a_{2}-T_{2} x\right) / 2+\left(I_{0}^{2}-2 I_{0} T_{0}\right) / 4\left(a_{0}-T_{0} x\right)^{2}$,

$$
\begin{aligned}
\phi_{2}(x)= & b_{2}+\frac{I_{2} T_{0}-I_{0} T_{2}}{T_{0}^{2}} \ln \left|a_{0}-T_{0} x\right| \\
& +\frac{I_{0}\left(a_{2} T_{0}-a_{0} T_{2}\right)}{T_{0}^{2}\left(a_{0}-T_{0} x\right)}+\frac{I_{0}\left(I_{0}^{2}-4 T_{0}^{2}\right)}{6 T_{0}\left(a_{0}-T_{0} x\right)^{3}} .
\end{aligned}
$$

For the inner system, we have

- At the boundary $x=0$ with $x=\varepsilon \xi$,

- Zeroth order: $\Phi_{0}(\xi)=\bar{V}, U_{0}(\xi)=0, C_{10}(\xi)=C_{20}(\xi)=$ $L$.

- First order: $\Phi_{1}(\xi)=-I_{0} / 2 L \xi, C_{11}(\xi)=C_{21}(\xi)=$ $-T_{0} / 2 \xi$

- Second order: $C_{12}(\xi)=\left(I_{0} T_{0}\right) / 8 L^{2}\left(1-e^{-\sqrt{2 L} \xi}\right), C_{22}(\xi)=$ $\left(I_{0} T_{0}\right) / 8 L^{2}\left(e^{-\sqrt{2 L} \xi}-1\right), \Phi_{2}(\xi)=\left(I_{0} T_{0}\right) / 8 L^{3}\left(e^{-\sqrt{2 L} \xi}-\right.$ 1) $-I_{0} T_{0} / 8 L^{2} \xi^{2}$.

- At the boundary $x=1$ with $x-1=\varepsilon \xi$,

- Zeroth order: $\Psi_{0}(\xi)=0, V_{0}(\xi)=0, D_{10}(\xi)=D_{20}(\xi)=$ $R$.

- First order: $\Psi_{1}(\xi)=-I_{0} / 2 R \xi, D_{11}(\xi)=D_{21}(\xi)=$ $-T_{0} / 2 \xi$.

- Second order: $D_{12}(\xi)=-I_{0} T_{0} / 8 R^{2}\left(e^{\sqrt{2 R} \xi}-1\right), D_{22}(\xi)=$ $I_{0} T_{0} / 8 R^{2}\left(e^{\sqrt{2 R} \xi}-1\right), \Psi_{2}(\xi)=I_{0} T_{0} / 8 R^{3}\left(e^{\sqrt{2 R} \xi}-1\right)-$ $I_{0} T_{0} / 8 R^{2} \xi^{2}$. 
Here,

$$
\begin{aligned}
a_{0}= & 2 L, \quad T_{0}=2(L-R), \quad I_{0}=\frac{2(L-R)}{\ln L-\ln R} \bar{V}, \quad b_{0}=\bar{V}-\frac{I_{0}}{T_{0}} \ln 2 L ; \\
a_{1}= & I_{1}=T_{1}=b_{1}=0 ; \quad a_{2}=-\frac{I_{0}^{2}}{8 L^{2}}, \\
T_{2}= & \frac{(L-R)^{3}(L+R)}{2 L^{2} R^{2}(\ln L-\ln R)^{2}} \bar{V}^{2}, \\
I_{2}= & \frac{(L-R)^{3}\left(L^{3}-R^{3}\right) \bar{V}}{3 L^{3} R^{3}(\ln L-\ln R)^{2}} \\
& +\frac{(L-R)^{3}}{L^{2} R^{2}(\ln L-\ln R)^{3}}\left(\frac{L+R}{2}-\frac{L^{3}-R^{3}}{3 L R(\ln L-\ln R)}\right) \bar{V}^{3}, \\
b_{2}= & \frac{I_{0}\left(4 T_{0}^{2}-I_{0}^{2}\right)}{6 a_{0}^{3} T_{0}}-\frac{I_{0}\left(a_{2} T_{0}-a_{0} T_{2}\right)}{a_{0} T_{0}^{2}} \\
& -\frac{I_{2} T_{0}-I_{0} T_{2}}{T_{0}^{2}} \ln \left|a_{0}\right|-\frac{I_{0} T_{0}}{8 L^{3}} .
\end{aligned}
$$

Now we carry out the analysis for the third order asymptotic expansions and matchings in detail.

3.1. Third order outer expansion. The third order outer system, from $(2.2)$, is

$$
\begin{aligned}
& \ddot{\phi}_{1}=-c_{13}+c_{23}, \quad \dot{c}_{13}=-\left(c_{13} \dot{\phi}_{0}+c_{12} \dot{\phi}_{1}+c_{11} \dot{\phi}_{2}+c_{10} \dot{\phi}_{3}\right)-J_{13}, \\
& \dot{c}_{23}=\left(c_{23} \dot{\phi}_{0}+c_{22} \dot{\phi}_{1}+c_{21} \dot{\phi}_{2}+c_{20} \dot{\phi}_{3}\right)-J_{23} .
\end{aligned}
$$

Solving (3.1), together with Theorem 3.1, we have

$$
\begin{aligned}
c_{13}(x)= & c_{23}(x)=\frac{a_{3}-T_{3} x}{2}, \\
\phi_{3}(x)= & b_{3}+\left(\frac{a_{3} I_{0}}{T_{0}}-\frac{a_{0} I_{0} T_{3}}{T_{0}^{2}}\right) \frac{1}{a_{0}-T_{0} x} \\
& +\left(\frac{I_{3}}{T_{0}}-\frac{I_{0} T_{3}}{T_{0}^{2}}\right) \ln \left|a_{0}-T_{0} x\right|,
\end{aligned}
$$

for some constants $a_{3}$ and $b_{3}$ to be determined through matching. Here $I_{3}=J_{13}-J_{23}$ and $T_{3}=J_{13}+J_{23}$. 
3.2. Third order inner expansion. At the boundary $x=0$, from (2.5), the third order inner system is

$$
\begin{aligned}
\Phi_{3}^{\prime} & =U_{3}, \quad U_{3}^{\prime}=-\left(C_{13}-C_{23}\right), \\
C_{13}^{\prime} & =-\left(C_{10} U_{3}+C_{11} U_{2}+C_{12} U_{1}+C_{13} U_{0}\right)-J_{12}, \\
C_{23}^{\prime} & =\left(C_{20} U_{3}+C_{21} U_{2}+C_{22} U_{1}+C_{23} U_{0}\right)-J_{22} .
\end{aligned}
$$

As an application of Proposition 2.1 for zeroth, first and second order cases (see [1, Propositions 3.1, 3.2, 3.3] and Proposition 2.2), we have the next result.

Proposition 3.2. System (3.3) has the following integrals:

$$
\begin{aligned}
& G_{1}=C_{13} e^{\Phi_{0}}+C_{10} e^{\Phi_{0}} \Phi_{3}+J_{12} F_{1}+F_{131}+F_{132}, \\
& G_{2}=C_{23} e^{-\Phi_{0}}-C_{20} e^{-\Phi_{0}} \Phi_{3}+J_{22} F_{2}-F_{231}-F_{232}, \\
& G_{3}=U_{0} U_{3}+U_{1} U_{2}-C_{13}-C_{23}-T_{2} \xi,
\end{aligned}
$$

where

$$
\begin{aligned}
F_{1}(\xi) & =\int_{0}^{\xi} e^{\Phi_{0}(s)} d s, \\
F_{2}(\xi) & =\int_{0}^{\xi} e^{-\Phi_{0}(s)} d s, \\
F_{131}(\xi) & =\int_{0}^{\xi} C_{11}(s) U_{2}(s) e^{\Phi_{0}(s)} d s, \\
F_{132}(\xi) & =\int_{0}^{\xi} C_{12}(s) U_{1}(s) e^{\Phi_{0}(s)} d s, \\
F_{231}(\xi) & =\int_{0}^{\xi} C_{21}(s) U_{2}(s) e^{-\Phi_{0}(s)} d s, \\
F_{232}(\xi) & =\int_{0}^{\xi} C_{22}(s) U_{1}(s) e^{-\Phi_{0}(s)} d s .
\end{aligned}
$$

Proof. This can be verified directly.

Applying the integrals in Proposition 3.2, we can solve (3.3) with $\Phi_{3}(0)=C_{13}(0)=C_{23}(0)=0$ to get

$$
\Phi_{3}(\xi)=\left[\frac{I_{0} T_{0}^{2}}{2(2 L)^{7 / 2}}\left(\frac{1}{2} \xi^{2}+\frac{3}{2 \sqrt{2 L}} \xi+\frac{1}{L}\right)-\gamma_{1}\right] e^{-\sqrt{2 L} \xi}+\gamma_{1} e^{\sqrt{2 L} \xi}
$$




$$
-\frac{I_{0} T_{0}^{2}}{(2 L)^{\frac{9}{2}}}-\left(\frac{I_{2}}{2 L}+\frac{2 I_{0} T_{0}^{2}}{(2 L)^{4}}\right) \xi-\frac{I_{0} T_{0}^{2}}{3(2 L)^{3}} \xi^{3} .
$$

The matching will force $\gamma_{1}=0$. For convenience, we define the following functions.

$$
\begin{aligned}
& k_{1}(x)=\frac{I_{0} T_{0}^{2}}{2(2 x)^{7 / 2}}\left(\frac{1}{2} \xi^{2}+\frac{3}{2 \sqrt{2 x}} \xi+\frac{1}{x}\right), \\
& k_{2}(x)=\frac{I_{0} T_{0}}{4(2 x)^{5 / 2}}\left(\frac{I_{0}}{x}-\frac{T_{0}}{2 \sqrt{2 x}} \xi-\frac{T_{0}}{2} \xi^{2}\right), \\
& k_{3}(x)=\frac{I_{0} T_{0}}{4(2 x)^{5 / 2}}\left(\frac{I_{0}}{x}+\frac{T_{0}}{2 \sqrt{2 x}} \xi+\frac{T_{0}}{2} \xi^{2}\right) .
\end{aligned}
$$

Then, for $\xi \geq 0$,

$$
\begin{gathered}
\Phi_{3}(\xi)=k_{1}(L) e^{-\sqrt{2 L} \xi}-\frac{I_{0} T_{0}^{2}}{(2 L)^{9 / 2}}-\left(\frac{I_{2}}{2 L}+\frac{2 I_{0} T_{0}^{2}}{(2 L)^{4}}\right) \xi-\frac{I_{0} T_{0}^{2}}{3(2 L)^{3}} \xi^{3}, \\
C_{13}(\xi)=k_{2}(L) e^{-\sqrt{2 L} \xi}+\left(\frac{I_{0} T_{0}\left(I_{0}+2 T_{0}\right)}{16 L^{3}}-\frac{T_{2}}{2}\right) \xi-\frac{I_{0}^{2} T_{0}}{2(2 L)^{7 / 2}}, \\
C_{23}(\xi)=k_{3}(L) e^{-\sqrt{2 L} \xi}+\left(\frac{I_{0} T_{0}\left(I_{0}-2 T_{0}\right)}{16 L^{3}}-\frac{T_{2}}{2}\right) \xi-\frac{I_{0}^{2} T_{0}}{2(2 L)^{7 / 2}} .
\end{gathered}
$$

Similarly, at $x=1$, the third order inner solution is, for $\xi \leq 0$,

$$
\begin{aligned}
\Psi_{3}(\xi)= & -k_{1}(R) e^{\sqrt{2 R} \xi}+\frac{I_{0} T_{0}^{2}}{(2 R)^{9 / 2}} \\
& -\left(\frac{I_{2}}{2 R}+\frac{2 I_{0} T_{0}^{2}}{(2 R)^{4}}\right) \xi-\frac{I_{0} T_{0}^{2}}{3(2 R)^{3}} \xi^{3} \\
D_{13}(\xi)= & -k_{2}(R) e^{\sqrt{2 R} \xi} \\
& +\left(\frac{I_{0} T_{0}\left(I_{0}+2 T_{0}\right)}{16 R^{3}}-\frac{T_{2}}{2}\right) \xi+\frac{I_{0}^{2} T_{0}}{2(2 R)^{7 / 2}}, \\
D_{23}(\xi)= & -k_{3}(R) e^{\sqrt{2 R} \xi}+\left(\frac{I_{0} T_{0}\left(I_{0}-2 T_{0}\right)}{16 R^{3}}-\frac{T_{2}}{2}\right) \xi \\
& +\frac{I_{0}^{2} T_{0}}{2(2 R)^{7 / 2}} .
\end{aligned}
$$


3.3. Third order matching. For convenience, we define

$$
\begin{aligned}
\rho_{1}(x)= & b_{2}-\frac{I_{0} T_{0}}{2\left(a_{0}-x\right)^{2}} \xi^{2}+\frac{I_{2} T_{0}-I_{0} T_{2}}{T_{0}^{2}} \ln \left|a_{0}-x\right| \\
& +\frac{I_{0}\left(a_{2} T_{0}-a_{0} T_{2}\right)}{T_{0}^{2}\left(a_{0}-x\right)}-\frac{I_{0}\left(4 T_{0}^{2}-I_{0}^{2}\right)}{6 T_{0}\left(a_{0}-x\right)^{3}}, \\
\rho_{2}(x)= & b_{3}+\left(\frac{I_{0}\left(I_{0}^{2}-4 T_{0}^{2}\right)}{2\left(a_{0}-x\right)^{4}}+\frac{\left(a_{2}-T_{2}\right) I_{0}}{\left(a_{0}-x\right)^{2}}-\frac{I_{2}}{a_{0}-x}\right) \xi \\
& -\frac{I_{0} T_{0}^{2}}{3\left(a_{0}-x\right)^{3}} \xi^{3}+\frac{a_{3} I_{0} T_{0}-a_{0} I_{0} T_{3}}{T_{0}^{2}\left(a_{0}-x\right)} \\
& +\frac{I_{3} T_{0}-I_{0} T_{3}}{T_{0}^{2}} \ln \left|a_{0}-x\right|, \\
\rho_{3}(x, y)= & \frac{a_{3}-y}{2}+\left(\frac{I_{0}^{2} T_{0}}{2\left(a_{0}-x\right)^{3}}+\frac{I_{0} T_{0}^{2}}{\left(a_{0}-x\right)^{3}}-\frac{T_{2}}{2}\right) \xi, \\
\rho_{4}(x, y)= & \frac{a_{3}-y}{2}+\left(\frac{I_{0}^{2} T_{0}}{2\left(a_{0}-x\right)^{3}}-\frac{I_{0} T_{0}^{2}}{\left(a_{0}-x\right)^{3}}-\frac{T_{2}}{2}\right) \xi .
\end{aligned}
$$

From (3.2) and (3.6), in terms of $\xi=x / \varepsilon$, the outer expansion at $x=0$ is

$$
\begin{aligned}
E_{\xi}^{3} E_{x}^{3}(\phi)= & b_{0}+\frac{I_{0}}{T_{0}} \ln a_{0}-\varepsilon \frac{I_{0}}{a_{0}} \xi+\varepsilon^{2} \rho_{1}(0)+\varepsilon^{3} \rho_{2}(0), \\
E_{\xi}^{3} E_{x}^{3}\left(c_{1}\right)= & \frac{a_{0}}{2}-\varepsilon \frac{T_{0}}{2} \xi+\varepsilon^{2}\left(\frac{a_{2}}{2}+\frac{I_{0}^{2}+2 I_{0} T_{0}}{4 a_{0}}\right) \\
& +\varepsilon^{3} \rho_{3}(0,0) \\
E_{\xi}^{3} E_{x}^{3}\left(c_{2}\right)= & \frac{a_{0}}{2}-\varepsilon \frac{T_{0}}{2} \xi+\varepsilon^{2}\left(\frac{a_{2}}{2}+\frac{I_{0}^{2}-2 I_{0} T_{0}}{4 a_{0}}\right) \\
& +\varepsilon^{3} \rho_{4}(0,0)
\end{aligned}
$$

and, in terms of $\xi=(x-1) / \varepsilon$, the outer expansion at $x=1$ is

$$
\begin{aligned}
E_{\xi}^{3} E_{x}^{3}(\phi)= & b_{0}+\frac{I_{0}}{T_{0}} \ln \left|a_{0}-T_{0}\right| \\
& -\varepsilon \frac{I_{0}}{a_{0}-T_{0}} \xi+\varepsilon^{2} \rho_{1}\left(T_{0}\right)+\varepsilon^{3} \rho_{2}\left(T_{0}\right) \\
E_{\xi}^{3} E_{x}^{3}\left(c_{1}\right)= & \frac{a_{0}-T_{0}}{2}-\varepsilon \frac{T_{0}}{2} \xi+\varepsilon^{2}\left(\frac{a_{2}-T_{2}}{2}+\frac{I_{0}^{2}+2 I_{0} T_{0}}{4\left(a_{0}-T_{0}\right)^{2}}\right)
\end{aligned}
$$




$$
\begin{aligned}
& +\varepsilon^{3} \rho_{3}\left(T_{0}, T_{3}\right), \\
E_{\xi}^{3} E_{x}^{3}\left(c_{2}\right)= & \frac{a_{0}-T_{0}}{2}-\varepsilon \frac{T_{0}}{2} \xi+\varepsilon^{2}\left(\frac{a_{2}-T_{2}}{2}+\frac{I_{0}^{2}-2 I_{0} T_{0}}{4\left(a_{0}-T_{0}\right)^{2}}\right) \\
& +\varepsilon^{3} \rho_{4}\left(T_{0}, T_{3}\right) .
\end{aligned}
$$

From (3.4) and (3.5), the inner expansion at $x=0$ is

$$
\begin{aligned}
E_{x}^{3} E_{\xi}^{3}(\Phi)= & \nu_{0}-\varepsilon \frac{I_{0}}{2 L} \xi-\varepsilon^{2}\left(\frac{I_{0} T_{0}}{8 L^{3}}+\frac{I_{0} T_{0}}{8 L^{2}} \xi^{2}\right) \\
& -\varepsilon^{3}\left(\frac{I_{0} T_{0}^{2}}{(2 L)^{9 / 2}}+\left(\frac{I_{2}}{2 L}+\frac{2 I_{0} T_{0}^{2}}{(2 L)^{4}}\right) \xi+\frac{I_{0} T_{0}^{2}}{3(2 L)^{3}} \xi^{3}\right), \\
E_{x}^{3} E_{\xi}^{3}\left(C_{1}\right)= & L-\varepsilon \frac{T_{0}}{2} \xi+\varepsilon^{2} \frac{I_{0} T_{0}}{8 L^{2}} \\
& -\varepsilon^{3}\left(\frac{I_{0}^{2} T_{0}}{2(2 L)^{7 / 2}}-\left(\frac{I_{0} T_{0}^{2}}{8 L^{3}}+\frac{I_{0}^{2} T_{0}}{16 L^{3}}-\frac{T_{2}}{2}\right) \xi\right), \\
E_{x}^{3} E_{\xi}^{3}\left(C_{2}\right)= & L-\varepsilon \frac{T_{0}}{2} \xi-\varepsilon^{2} \frac{I_{0} T_{0}}{8 L^{2}} \\
& -\varepsilon^{3}\left(\frac{I_{0}^{2} T_{0}}{2(2 L)^{7 / 2}}-\left(\frac{I_{0}^{2} T_{0}}{16 L^{3}}-\frac{I_{0} T_{0}^{2}}{8 L^{3}}-\frac{T_{2}}{2}\right) \xi\right),
\end{aligned}
$$

and the inner expansion at $x=1$ is

$$
\begin{aligned}
E_{x}^{3} E_{\xi}^{3}(\Psi)= & \nu_{0}-\varepsilon \frac{I_{0}}{2 R} \xi-\varepsilon^{2}\left(\frac{I_{0} T_{0}}{8 R^{3}}+\frac{I_{0} T_{0}}{8 R^{2}} \xi^{2}\right) \\
& -\varepsilon^{3}\left(-\frac{I_{0} T_{0}^{2}}{(2 R)^{9 / 2}}+\left(\frac{I_{2}}{2 R}+\frac{2 I_{0} T_{0}^{2}}{(2 R)^{4}}\right) \xi+\frac{I_{0} T_{0}^{2}}{3(2 R)^{3}} \xi^{3}\right), \\
E_{x}^{3} E_{\xi}^{3}\left(D_{1}\right)= & R-\varepsilon \frac{T_{0}}{2} \xi+\varepsilon^{2} \frac{I_{0} T_{0}}{8 R^{2}} \\
& +\varepsilon^{3}\left(\frac{I_{0}^{2} T_{0}}{2(2 R)^{7 / 2}}+\left(\frac{I_{0}^{2} T_{0}}{16 R^{3}}+\frac{I_{0} T_{0}^{2}}{8 R^{3}}-\frac{T_{2}}{2}\right) \xi\right) \\
E_{x}^{3} E_{\xi}^{3}\left(D_{2}\right)= & R-\varepsilon \frac{T_{0}}{2} \xi-\varepsilon^{2} \frac{I_{0} T_{0}}{8 R^{2}} \\
& +\varepsilon^{3}\left(\frac{I_{0}^{2} T_{0}}{2(2 R)^{7 / 2}}+\left(\frac{I_{0}^{2} T_{0}}{16 R^{3}}-\frac{I_{0} T_{0}^{2}}{8 R^{3}}-\frac{T_{2}}{2}\right) \xi\right) .
\end{aligned}
$$

Together with Theorem 3.1, the matchings $E_{\xi}^{3} E_{x}^{3}(\phi)=E_{x}^{3} E_{\xi}^{3}(\Phi)$, $E_{\xi}^{3} E_{x}^{3}\left(c_{i}\right)=E_{x}^{3} E_{\xi}^{3}\left(C_{i}\right)$, at $x=0$ and $E_{\xi}^{3} E_{x}^{3}(\phi)=E_{x}^{3} E_{\xi}^{3}(\Psi), E_{\xi}^{3} E_{x}^{3}\left(c_{i}\right)=$ 
$E_{x}^{3} E_{\xi}^{3}\left(D_{i}\right)$ at $x=1$ for $i=1,2$, then give

$$
\begin{aligned}
a_{3}= & -\frac{I_{0}^{2} T_{0}}{(2 L)^{7 / 2}}, \quad T_{3}=-\frac{(L-R)^{3}}{\sqrt{2}(\ln L-\ln R)^{2}}\left(\frac{1}{R^{7 / 2}}+\frac{1}{L^{7 / 2}}\right) \bar{V}^{2}, \\
I_{3}= & -\frac{(L-R)^{4}}{\sqrt{2}(\ln L-\ln R)^{2}}\left(\frac{1}{R^{9 / 2}}+\frac{1}{L^{9 / 2}}\right) \bar{V} \\
& -\frac{(L-R)^{3}}{\sqrt{2}(\ln L-\ln R)^{3}} \\
& \times\left[\frac{1}{R^{7 / 2}}+\frac{1}{L^{7 / 2}}-\frac{L-R}{R L(\ln L-\ln R)}\left(\frac{L}{R^{7 / 2}}+\frac{R}{L^{7 / 2}}\right)\right] \bar{V}^{3}, \\
b_{3}= & \frac{I_{0} T_{3}-I_{3} T_{0}}{T_{0}^{2}} \ln \left|a_{0}\right|-\frac{I_{0}\left(a_{3} T_{0}-a_{0} T_{3}\right)}{a_{0} T_{0}^{2}}-\frac{I_{0} T_{0}^{2}}{(2 L)^{9 / 2}} .
\end{aligned}
$$

4. I-V relations under electro-neutrality conditions. Recall from (1.5) that, our main interest is to derive the asymptotic expansion of the I-V relation in the following form

$$
\mathcal{I}=I_{0}+\varepsilon I_{1}+\varepsilon^{2} I_{2}+\varepsilon^{3} I_{3}+\cdots .
$$

4.1. Main results. In this section, we will study the I-V relation under the electro-neutrality condition up to third order in $\varepsilon$ in detail, and state our main result.

From Theorem 3.1, and (3.7), under the assumption of electroneutrality, up to the third order in $\varepsilon$, we have

$$
\begin{aligned}
\mathcal{I} & =I_{0}+\varepsilon I_{1}+\varepsilon^{2} I_{2}+\varepsilon^{3} I_{3} \\
& =f(L, R, \varepsilon) \bar{V}-\varepsilon^{2} g(L, R, \varepsilon) \bar{V}^{3} \\
& =\frac{e}{k T}\left(f(L, R, \varepsilon) V-\varepsilon^{2}\left(\frac{e}{k T}\right)^{2} g(L, R, \varepsilon) V^{3}\right),
\end{aligned}
$$

where

$$
\begin{aligned}
f(L, R, \varepsilon)= & \frac{2(L-R)}{\ln L-\ln R} \\
& +\varepsilon^{2} \frac{(L-R)^{4}}{3(\ln L-\ln R)^{2}}\left[\frac{L^{2}+R^{2}+L R}{L^{3} R^{3}}-\frac{3 \varepsilon}{\sqrt{2}}\left(\frac{1}{L^{9 / 2}}+\frac{1}{R^{9 / 2}}\right)\right],
\end{aligned}
$$




$$
\begin{aligned}
g(L, R, \varepsilon)= & \frac{(L-R)^{3}\left(L^{3}-R^{3}\right)}{3 L^{3} R^{3}(\ln L-\ln R)^{4}}-\frac{(L-R)^{2}\left(L^{2}-R^{2}\right)}{2 L^{2} R^{2}(\ln L-\ln R)^{3}} \\
& +\varepsilon \frac{(L-R)^{3}}{\sqrt{2}(\ln L-\ln R)^{3}} \\
& \times\left[\frac{1}{R^{7 / 2}}+\frac{1}{L^{7 / 2}}-\frac{L-R}{R L(\ln L-\ln R)}\left(\frac{L}{R^{7 / 2}}+\frac{R}{L^{7 / 2}}\right)\right] .
\end{aligned}
$$

Theorem 4.1. If $L \neq R$, for $\varepsilon>0$ small, then, up to the order of $\varepsilon^{3}$, the $\mathrm{I}-\mathrm{V}$ relation $\mathcal{I}=\mathcal{I}(V)$ is a cubic function with three distinct real roots.

Proof. From (4.1), it suffices to show that both $f(L, R, \varepsilon)$ and $g(L, R, \varepsilon)$ are positive. Note that $(L-R) /(\ln L-\ln R)>0$, for $L \neq R$, our proof follows directly from the next three lemmas.

Lemma 4.2. For $L \neq R$, and $\varepsilon>0$ small,

$$
h_{1}(L, R, \varepsilon)=\frac{L^{2}+R^{2}+L R}{L^{3} R^{3}}-\frac{3 \varepsilon}{\sqrt{2}}\left(\frac{1}{L^{9 / 2}}+\frac{1}{R^{9 / 2}}\right)>0 .
$$

Proof. Treat $h_{1}(\varepsilon)=h_{1}(L, R, \varepsilon)$, for fixed $L \neq R$, one has $h_{1}\left(\varepsilon^{*}\right)=0, \quad$ and $\quad h_{1}^{\prime}(\varepsilon)=-\frac{3}{\sqrt{2}}\left(\frac{1}{R^{9 / 2}}+\frac{1}{L^{9 / 2}}\right)<0, \quad$ for all $\varepsilon>0$, where

$$
\varepsilon^{*}=\frac{\sqrt{2} L^{3 / 2} R^{3 / 2}\left(L^{2}+L R+R^{2}\right)}{3\left(L^{3 / 2}+R^{3 / 2}\right)\left(L^{3}+R^{3 / 2} L^{3 / 2}+R^{3}\right)} .
$$

It is clear that $h_{1}(\varepsilon)>0$ for $0<\varepsilon<\varepsilon^{*}$. Note that $\varepsilon \ll 1$ and $\varepsilon^{*}=O(1)$. We have $h_{1}(L, R, \varepsilon)>0$ for $\varepsilon>0$ small.

Lemma 4.3. For $L \neq R$,

$$
h_{2}(L, R)=\frac{(L-R)^{3}\left(L^{3}-R^{3}\right)}{3 L^{3} R^{3}(\ln L-\ln R)^{4}}-\frac{(L-R)^{2}\left(L^{2}-R^{2}\right)}{2 L^{2} R^{2}(\ln L-\ln R)^{3}}>0 .
$$


Proof. Noticing that $h_{2}(L, R)=h_{2}(R, L)$, it suffices to show that $h_{2}(L, R)>0$ for $L>R$. Rewrite $h_{2}(L, R)$ as

$$
h_{2}(L, R)=\frac{(L-R)^{3}}{L^{2} R^{2}(\ln L-\ln R)^{3}} \widetilde{h}_{2}(L, R),
$$

with

$$
\widetilde{h}_{2}(L, R)=\frac{L^{3}-R^{3}}{3 L R(\ln L-\ln R)}-\frac{L+R}{2} .
$$

Then $h_{2}(L, R)>0 \Leftrightarrow \widetilde{h}_{2}(L, R)>0$ for $L>R$. We fix $R$ by treating $\widetilde{h}_{2}(L)=\widetilde{h}_{2}(L, R)$ as a function of $L$. A direct calculation shows $\widetilde{h}_{2}(R)=\widetilde{h}_{2}^{\prime}(R)=0$, but $\widetilde{h}_{2}^{\prime \prime}(L)>0$ for all $L$. Therefore, we have $\widetilde{h}_{2}(L, R)>0$ for all $L>R$.

Lemma 4.4. For $L \neq R$,

$$
h_{3}(L, R, \varepsilon)=\frac{1}{R^{7 / 2}}+\frac{1}{L^{7 / 2}}-\frac{L-R}{R L(\ln L-\ln R)}\left(\frac{L}{R^{7 / 2}}+\frac{R}{L^{7 / 2}}\right)>0 .
$$

Proof. Rewrite $h_{3}(L, R)$ as $h_{3}(L, R)=p(L, R) /(L R)^{9 / 2}(\ln L-\ln R)$, where

$$
p(L, R)=L R(\ln L-\ln R)\left(L^{7 / 2}+R^{7 / 2}\right)-(L-R)\left(L^{9 / 2}+R^{9 / 2}\right) .
$$

Note that $h_{3}(L, R)=h_{3}(R, L)$. It suffices to show that $h_{3}(L, R)>0$ for $L>R$, which is equivalent to showing that $p(L, R)>0$ for $L>R$. To do so, we fix $R$ and treat $p(L)=p(L, R)$ as a function of $L$. Then, a direct computation gives $p(R)=p^{\prime}(R)=p^{\prime \prime}(R)=0$, but $p^{\prime \prime \prime}(L)>0$ for $L>R$. Therefore, $p(L)>0$ for $L>R$.

4.2. Remarks. For the third order terms, we only treated the electroneutrality case mainly because this is a natural biological assumption. Under this assumption, up to the order of $\varepsilon^{3}$, even though a quartic function is expected, the $\mathrm{I}-\mathrm{V}$ relation $\mathcal{I}(V)$ is still a cubic function with three distinct real roots, which is potentially related to the cubic-like feature of the average I-V relation of a population of channels in the Fitzhugh-Nagumo simplification of the Hodgkin-Huxley model. The existence of three distinct real roots of the I-V relation is responsible for the bi-stable structure in the FitzHugh-Nagumo system. 
Recall from [1] that the first order correction to the zeroth order linear I-V relation is quadratic without electro-neutrality condition, and we believe that the analysis for the first order terms in [1] can be applied to third order terms in this work without the electro-neutrality assumption.

For the fourth order correction to zeroth order I-V relation under the electro-neutrality condition, we have

Theorem 4.5. Under the electro-neutrality condition, we have

$$
\begin{aligned}
T_{4}= & \frac{3(L-R)^{4}\left(L^{5}-R^{5}\right)}{4 L^{5} R^{5}(\ln L-\ln R)} V \\
& +\frac{(L-R)^{4}}{2 L^{5} R^{5}(\ln L-\ln R)^{2}}\left(\frac{\left(L^{2}-R^{2}\right)\left(L^{3}-R^{3}\right)}{3(\ln L-\ln R)}+\frac{7\left(L^{5}-R^{5}\right)}{2}\right) V^{2} \\
& -\frac{(L-R)^{4}\left(L^{5}-R^{5}\right)}{4 L^{5} R^{5}(\ln L-\ln R)^{3}} V^{3}+\frac{(L-R)^{5}(L+R)}{2 L^{4} R^{4}(\ln L-\ln R)^{4}} \\
& \times\left(\frac{(L+R)}{2}-\frac{L^{3}-R^{3}}{3 L R(\ln L-\ln R)}\right) V^{4}, \\
I_{4}= & \frac{(L-R)^{4}}{L^{4} R^{4}(\ln L-\ln R)^{2}}\left(\frac{(L-R)\left(L^{3}-R^{3}\right) Q_{1}(L, R)}{6 L^{2} R^{2}} V\right. \\
& +\frac{3 Q_{3}(L, R)}{4 L R} V^{2}+\frac{Q_{2}(L, R)}{\ln L-\ln R} V^{3} \\
& \left.-\frac{Q_{3}(L, R)}{4 L R(\ln L-\ln R)^{2}} V^{4}+\frac{(L-R) Q_{4}(L, R)}{2(\ln L-\ln R)^{3}} V^{5}\right),
\end{aligned}
$$

where

$$
\begin{aligned}
Q_{1}(L, R)= & \frac{L^{3}-R^{3}}{3(\ln L-\ln R)}+\frac{97\left(L^{3}+R^{3}\right)}{2}, \\
Q_{2}(L, R)= & \frac{(L-R)\left(L^{5}-R^{5}\right)}{2 L^{2} R^{2}(\ln L-\ln R)}+\frac{(L-R)^{2}\left(L^{3}-R^{3}\right)\left(L^{2}+L R+R^{2}\right)}{9 L^{2} R^{2}(\ln L-\ln R)^{2}} \\
+ & \frac{13(L-R)\left(L^{5}-R^{5}\right)}{12 L^{2} R^{2}(\ln L-\ln R)}+\frac{7\left(L^{5}-R^{5}\right)}{4 L R}-\frac{(L-R)^{2}(L+R)}{2(\ln L-\ln R)} \\
& +\frac{(L-R)(L+R)\left(L^{3}-R^{3}\right)}{4 L R(\ln L-\ln R)}-\frac{17(L-R)^{2}}{12(\ln L-\ln R)},
\end{aligned}
$$




$$
\begin{aligned}
Q_{3}(L, R)= & \frac{(L-R)\left(L^{5}-R^{5}\right)}{\ln L-\ln R}-\frac{(L-R)^{2}}{\ln L-\ln R}+L^{5}-R^{5}, \\
Q_{4}(L, R)= & \frac{(L+R)\left(L^{3}-R^{3}\right)}{2 L R(\ln L-\ln R)}-\frac{\left(L^{3}-R^{3}\right)^{2}}{3 L^{2} R^{2}(\ln L-\ln R)^{2}} \\
& +\frac{3(L+R)^{2}}{4} .
\end{aligned}
$$

Remark 4.6. Under the electro-neutrality condition, up to the fourth order in $\varepsilon$, the I-V relation function $\mathcal{I}(V)$ is quintic instead of being cubic. However, for $\varepsilon>0$ small, $\mathcal{I}=I_{0}+\varepsilon I_{1}+\varepsilon^{2} I_{2}+\varepsilon^{3} I_{3}$ is good enough to approximate the I-V relation, which can be seen from last section.

To end this section, we have the following interesting result about the I-V relations, which can be checked directly from systems (1.7) and (1.8).

Proposition 4.7. For all $\varepsilon>0$ small, $\mathcal{I}(L, R, \bar{V} ; \varepsilon)=-\mathcal{I}(R, L,-\bar{V} ; \varepsilon)$. $A$ direct observation shows that, for $j=0,1,2,3,4$,

$$
\begin{aligned}
I_{j}(L, R, V ; 0) & =-I_{j}(R, L,-V ; 0), \\
T_{j}(L, R, V ; 0) & =-T_{j}(R, L,-V ; 0) .
\end{aligned}
$$

5. Numerical simulations. In this section, numerical simulations are performed to system (1.7) with the boundary condition (1.8) to check the cubic-like feature of the I-V Curve and investigate the effects of the boundary conditions, the permanent charge on the I-V relations.

5.1. BVP solver for (1.7) and (1.8) and initial guess. In this section, we use bvp4c in Matlab ([11]) as the solver for our PNP system (1.7) and (1.8). It solves first order systems of ordinary differential equations with two-point boundary conditions in the following form:

$$
\left\{\begin{array}{l}
y^{\prime}=f(x, y) \quad a<x<b \\
g(y(a), y(b))=0 .
\end{array}\right.
$$

Given a mesh partition $a=x_{0}<x_{1}<\cdots<x_{k}=b$, the numerical solution of (5.1) is approximated by a piecewise cubic polynomial function $P(x)$. The approximated solution $P(x)$ satisfies the boundary 
conditions, and it is a cubic Hermite interpolation polynomial for each subinterval $\left[x_{i}, x_{i+1}\right]$.

For $i=0,1,2, \ldots, k-1$, let $y_{i}=P\left(x_{i}\right)$, and let $h_{i}=x_{i+1}-x_{i}$. The $y_{i}$ 's are evaluated by solving the algebraic equations

$$
\Phi(X, Y)=\left(\phi_{0}(X, Y), \phi_{1}(X, Y), \ldots, \phi_{k}(X, Y)\right)=0
$$

where

$$
\begin{aligned}
X & =\left[x_{0}, x_{1}, \cdots, x_{k}\right]^{T}, \\
Y & =\left[y_{0}, y_{1}, \cdots, y_{k}\right]^{T}, \\
\phi_{0}(X, Y) & =g\left(y_{0}, y_{k}\right), \\
\phi_{i}(X, Y) & =y_{i}-y_{i-1}-\frac{1}{6} h_{i-1}\left(f_{i-1}+4 f_{i}^{*}+f_{i}\right), \quad i=1,2, \ldots, k,
\end{aligned}
$$

and

$$
\begin{aligned}
f_{i} & =f\left(x_{i}, y_{i}\right) \\
f_{i}^{*} & =f\left(\frac{1}{2}\left(x_{i-1}+x_{i}\right), \frac{1}{2}\left(y_{i-1}+y_{i}\right)-\frac{1}{8} h_{i-1}\left(f_{i}-f_{i-1}\right)\right) .
\end{aligned}
$$

The algebraic system (5.2) is solved by the simplified Newton's method with a weak line search. The global Jacobian $\partial \Phi / \partial Y$ (using finite difference approximation by default) is required, and the structure of the Jacobian is important for the linear solver in each Newton's iteration. The residual of $P(x)$ is calculated by $r(x)=P(x)-$ $f(x, P(x))$, and the residual in the boundary condition is $g(P(a), P(b))$. The adaptive mesh strategy has been used to control the residual in "bvp4c," for details, see [11].

To apply bvp4c, we first rewrite (1.7) into a system of first order equations as

$$
\begin{aligned}
& \varepsilon \frac{d}{d x} \phi=u, \\
& \frac{\varepsilon}{h(x)} \frac{d}{d x}(h(x) u)=-\left(\alpha c_{1}-\beta c_{2}+Q(x)\right), \\
& \frac{d J_{i}}{d x}=0 \\
& \varepsilon h(x) \frac{d c_{1}}{d x}+\alpha h(x) c_{1} u=-\varepsilon J_{1},
\end{aligned}
$$




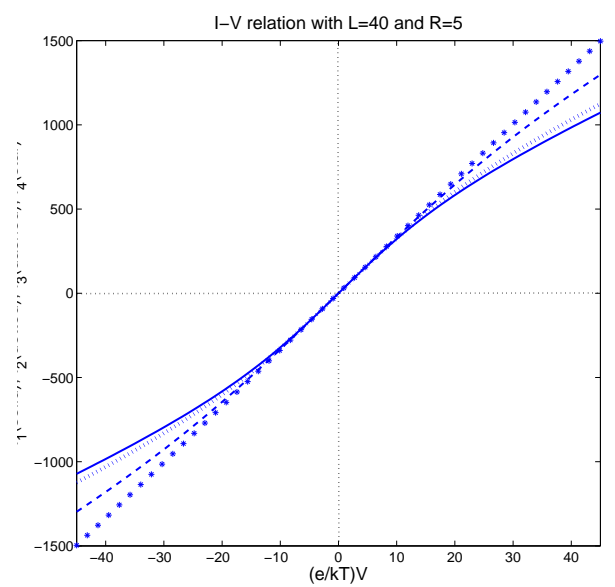

FIGURE 1. Numerical simulation of the I-V relation $\mathcal{I}(V)$ with $Q=$ 0 . $\mathcal{I}_{1}$ (solid curve, $\left.\varepsilon=0.1\right), \mathcal{I}_{2}($ dotted curve, $\varepsilon=0.08), \mathcal{I}_{3}($ dashed curve, $\varepsilon=0.04)$, and $\mathcal{I}_{4}($ stars, $\varepsilon=0.008)$

$$
\varepsilon h(x) \frac{d c_{2}}{d x}-\beta h(x) c_{2} u=-\varepsilon J_{2},
$$

with the same boundary condition (1.8).

For a general iteration step, we take the initial guess from the approximate solution of the previous fixed point iteration. At the first iteration, for the case where $Q=0$, we take advantage of the analysis from [1] and choose the initial guess $\left(\phi^{0}, u^{0}, c_{1}^{0}, c_{2}^{0}, J_{1}^{0}, J_{2}^{0}\right)$ as follows.

We take the zeroth order outer solution from [1] as our initial guess for both $Q(x)=0$ and $Q(x) \neq 0$ :

$$
\begin{aligned}
\phi_{0}^{0}(x) & =\frac{\ln |L-(L-R) x|-\ln R}{\ln L-\ln R} \nu_{0}, \\
u_{0}^{0}(x) & =\frac{(L-R) \nu_{0}}{(\ln L-\ln R)((L-R) x-L)}, \\
c_{10}^{0}(x) & =c_{20}^{0}(x)=L-(L-R) x, \\
J_{10}^{0} & =(L-R)\left(1+\frac{\nu_{0}}{\ln L-\ln R}\right),
\end{aligned}
$$



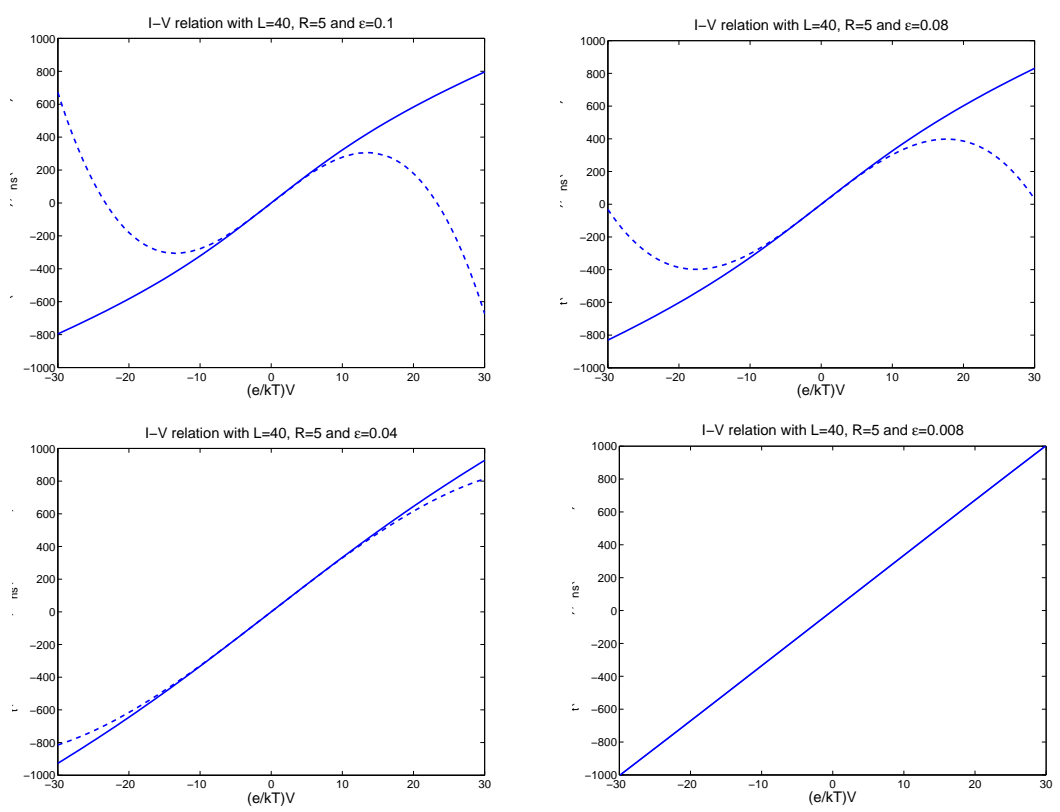

Figure 2. Plots of $\mathcal{I}(V)$ as a function of $V$ for $Q=0$ with $L=40$, $R=5$ and $\varepsilon=0.1,0.08,0.04$ and 0.008 , respectively. $\mathcal{I}_{t}$-the third order approximation, and $\mathcal{I}_{n s}$-from numerical simulation.

$$
J_{20}^{0}=(L-R)\left(1-\frac{\nu_{0}}{\ln L-\ln R}\right) .
$$

We take a uniform mesh partition as an initial mesh and evaluate the functions $\left(\phi_{0}^{0}, u_{0}^{0}, c_{10}^{0}, c_{20}^{0}, J_{10}^{0}, J_{20}^{0}\right)$ at these mesh points as an initial guess for bvp4c at our first fixed point iteration. We use the mesh and solution from the previous fixed point iteration as our initial mesh and initial guess for the late iteration.

5.2. Numerical experiments. In this section, three numerical experiments are conducted to system (5.3) with boundary conditions (1.8), respectively, which are stated as follows:

- Experiment 1: for $Q(x)=0$, fixing $L$ and $R$, letting $\varepsilon$ vary, we check the cubic-like feature of the I-V relation, and, meanwhile, 


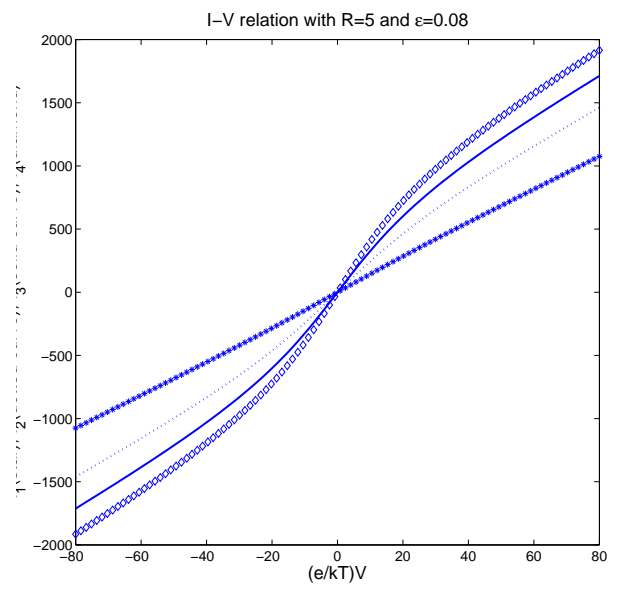

FIGURE 3. Numerical simulation of the I-V relation $\mathcal{I}(V)$ with $Q=0$. $\mathcal{I}_{1}$ (stars, $L=10), \mathcal{I}_{2}$ (dotted curve, $L=25$ ), $\mathcal{I}_{3}$ (solid curve, $L=40$ ) and $\mathcal{I}_{4}$ (diamonds, $L=55$ ).

compare the I-V curves from numerical simulation with the ones obtained from asymptotic expansions;

- Experiment 2: for $Q(x)=0$, fixing $R$ and $\varepsilon$, letting $L$ vary, we investigate the effect of the concentration boundary condition on the I-V curve;

- Experiment 3: for $Q=\left\{\begin{array}{l}0 \quad 0 \leq x<a, \\ Q_{0} a \leq x \leq b, \\ 0 \quad b<x \leq 1,\end{array}\right.$ fixing $L, R$ and $\varepsilon$, letting $Q_{0}$ vary, we investigate the effect of the permanent charge on the I-V relation curve and check the cubic-like feature of the I-V curve.

For Experiment 1, the following properties are predicted from the analytical results and can be observed from the numerical simulations: For the first part, we have (see Figure 1)

(i) all I-V curves pass through the point $(0,0)$ and, for $V$ close to 0 , the value of $\varepsilon$ has less effect on the I-V curve;

(ii) for $V>0$, the I-V curve is decreasing in $\varepsilon$ and, for $V<0$, the 

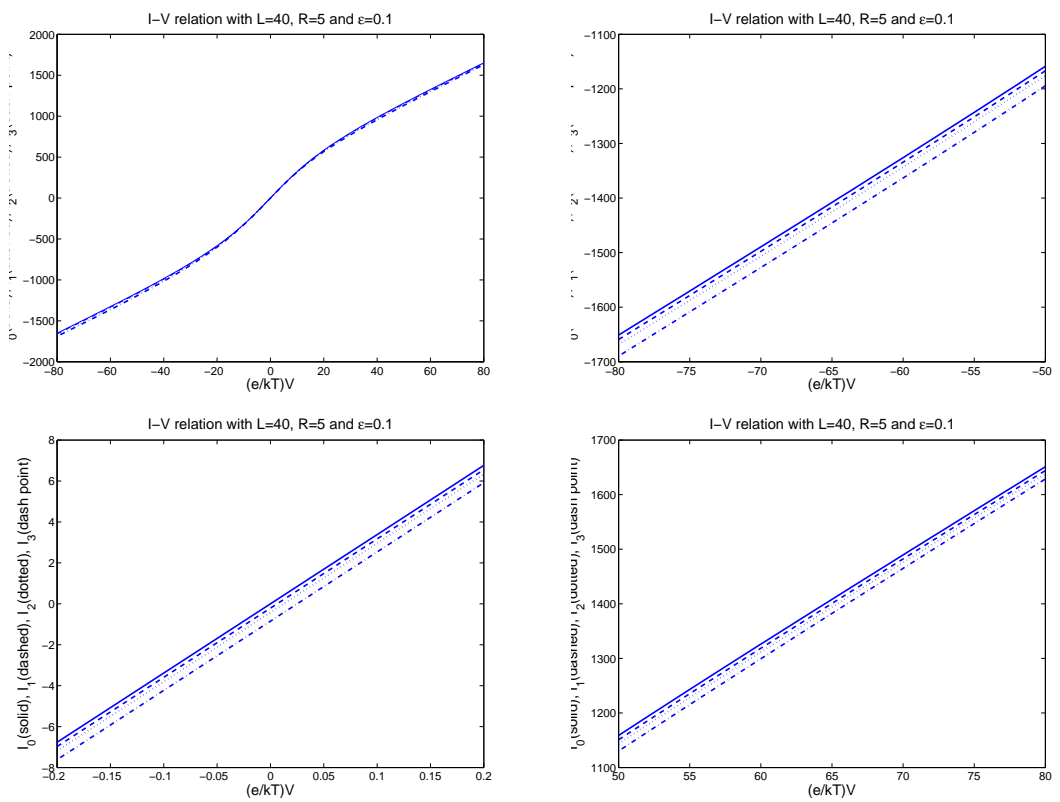

Figure 4. Plots of $I(V)$ as a function of $V$ for $L=40, R=5$ and $\varepsilon=0.1$, with $Q=0,0.05,0.1$ and 0.2 , respectively. The left graph in the first row is the simulation over the interval $[-80,80]$; for the other three graphs, we focus on different subintervals. $\mathcal{I}_{0}$ (solid curve) with $Q_{0}=0, \mathcal{I}_{1}$ (dashed curve) with $Q_{0}=0.05, \mathcal{I}_{2}$ (dotted curve) with $Q_{0}=0.1$ and $\mathcal{I}_{3}$ (dash point) with $Q_{0}=0.2$.

$\mathrm{I}-\mathrm{V}$ curve is increasing in $\varepsilon$;

(iii) the I-V curve is more cubic-like for larger $\varepsilon>0$ and, for $\varepsilon$ small enough, the $\mathrm{I}-\mathrm{V}$ relation curve $\mathcal{I}(V)$ is close to the zeroth order approximation $\mathcal{I}_{0}=2(L-R) V /(\ln L-\ln R)$ under the electroneutrality condition.

For the second part, one has (see Figure 2)

(i) the smaller $\varepsilon$ is, the better approximation $\mathcal{I}_{t}$ (the third order approximation to the I-V curve) will be;

(ii) the approximation is sensitive to $V$, for $V$ close to 0 , the value of $\varepsilon$ has less effect on the approximation. 
For Experiment 2, the following properties can be observed from the numerical simulations (see Figure 3):

(i) all curves pass the point $(0,0)$, for $V>0$, the I-V curves increase in $L$, and, for $V<0$, they are decreasing in $L$;

(ii) for fixed $\varepsilon$ and $R$, the I-V curve is more cubic-like for larger difference $L-R$.

For experiment 3, we investigate:

(i) all curves pass through the point $(0,0)$, and the I-V curves still keep the cubic-like feature;

(ii) the I-V curves decrease in the permanent charge $Q_{0}$ (see Figure $4)$.

Acknowledgments. This work was initiated from a private talk with Dr. Nicole Abaid and Dr. Bob Eisenberg, and encouraged by Dr. Weishi Liu. The author thanks Dr. Weishi Liu for introducing such an interesting field and much encouragement.

\section{REFERENCES}

1. N. Abaid, R.S. Eisenberg and W. Liu, Asymptotic expansions of $\mathrm{I}-\mathrm{V}$ relations via Poisson-Nernst-Planck system, SIAM J. Appl. Dynam. Syst. 7 (2008), 15071526.

2. V. Barcilon, Ion flow through narrow membrane channels: Part I, SIAM J. Appl. Math. 52 (1992), 1391-1404.

3. V. Barcilon, D.-P. Chen and R.S. Eisenberg, Ion flow through narrow membrane channels: Part II, SIAM J. Appl. Math. 52 (1992), 1405-1425.

4. V. Barcilon, D.-P. Chen, R.S. Eisenberg and J.W. Jerome, Qualitative properties of steady-state Poisson-Nernst-Planck systems: Perturbation and simulation study, SIAM J. Appl. Math. 57 (1997), 631-648.

5. W. Eckhaus, Asymptotic analysis of singular perturbations, Stud. Math. Appl. 9, North-Holland Publishing Co., Amsterdam, 1979.

6. _ Fundamental concepts of matching, SIAM Rev. 36 (1994), 431-439.

7. B. Eisenberg, Ionic channels in biological membranes: Electrostatic analysis of a natural nanotube, Contemp. Phys. 39 year? , 447-466.

8. B. Eisenberg and W. Liu, Poisson-Nernst-Planck systems for ion channels with permanent charges, SIAM J. Math. Anal. 38 (2007), 1932-1966.

9. D. Gillespie, A singular perturbation analysis of the Poisson-Nernst-Planck system: Applications to ionic channels, Ph.D dissertation, Rush University at Chicago, 1999. 
10. Shuguan Ji and Weishi Liu, Poisson-Nernst-Planck systems for ion flow with density functional theory for hard-sphere potential: I-V relations and critical potentials, Part I: Analysis, J. Dyn. Diff. Equat. 24 (2012), 955-983.

11. J. Kierzenka and L. Shampine, A BVP solver based on residual control and the Matlab PSE, ACM Trans. Math. Software 27 (2001), 299-316.

12. P.A. Lagerstrom, Matched asymptotic expansions, Springer-Verlag, New York, 1988.

13. G. Lin, W. Liu, Y. Yi and M. Zhang, Poisson-Nernst-Planck systems for ion flow with a local hard-sphere potential for ion sizes, SIAM J. Appl. Dynam. Syst. 12 (2013), 1613-1648.

14. W. Liu, One-dimensional steady-state Poisson-Nernst-Planck systems for ion channels with multiple ion species, J. Diff. Equat. 246 (2009), 428-451.

15. __ Geometric singular perturbation approach to steady-state PoissonNernst-Planck systems, SIAM J. Appl. Math. 65 (2005), 754-766.

16. W. Liu, X. Tu and M. Zhang, Poisson-Nernst-Planck Systems for ion flow with density functional theory for hard-sphere potential: I-V relations and critical potentials, Part II: Numerics, J. Dyn. Diff. Equat. 24 (2012), 985-1004.

17. W. Liu and B. Wang, Poisson-Nernst-Planck systems for narrow tubularlike membrane channels, J. Dynam. Diff. Equat. 22 (2010), 413-437.

18. M.S. Mock, An example of nonuniqueness of stationary solutions in device models, COMPEL 1 (1982), 165-174.

19. W. Nonner and R.S. Eisenberg, Ion permeation and glutamate residues linked by Poisson-Nernst-Planck theory in L-type calcium channels, Biophysical J. 75 (1998), 1287-1305.

20. R.E. O'Malley, Jr., Singular perturbation methods for ordinary differential equations, Appl. Math. Sci. 89, Springer-Verlag, New York, 1991.

21. I. Rubinstein, Multiple steady states in one-dimensonal electrodiffusion with local electroneutrality, SIAM J. Appl. Math. 47 (1987), 1076-1093.

22. phia, PA, 1990. , Electro-diffusion of ions, SIAM Stud. Appl. Math., SIAM, Philadel-

23. A. Singer, D. Gillespie, J. Norbury and R.S. Eisenberg, Singular perturbation analysis of the steady state Poisson-Nernst-Planck system: Applications to ion channels, Europ. J. Appl. Math. 19 (2008), 541-560.

24. A. Singer and J. Norbury, A Poisson-Nernst-Planck model for biological ion channels-An asymptotic analysis in a 3-D narrow funnel, SIAM J. Appl. Math. 70 (2009), 949-968.

25. H. Steinrück, Asymptotic analysis of the current-voltage curve of a pnpn semiconductor device, IMA J. Appl. Math. 43 (1989), 243-259.

26. A bifurcation analysis of the one-dimensional steady-state semiconductor device equations, SIAM J. Appl. Math. 49 (1989), 1102-1121. 
Department of Mathematics, New Mexico Institute of Mining and TechNOLOGY, SOCORRO, NM 87801

Email address: mzhang@nmt.edu, mzhang0129@gmail.com 